\title{
Multiple Format Search and Rescue Robot as a Competitive Arena
}

\author{
Andrew Chiou \\ School of Computing Sciences, CQUniversity Australia \\ Rockhampton Campus, 4702 QLD, Australia \\ a.chioulcqu.edu.au
}

\begin{abstract}
Current robot competition provides both students and researchers a platform to experiment with related technology and skills to solve challenging problems. However, the entry point for participation requires very demanding prerequisites of the participants. Even though robot competition is gaining popularity as a form of digital entertainment, it still remains a very specialised domain catering to academia. This paper presents on-going work in specifying, designing and developing a multiple format search and rescue robot to provide continuity for primary age participants to postgraduate researchers.
\end{abstract}

Keywords: robot, competition, search, rescue, arena.

\section{Introduction}

Competitive robots as entertainment and educational platform have been a popular domain in research. Several competitive formats have gained ever increasing followers, such as FIRA, RoboCup and RoboCup Jr. [1, 2]. The format of these competitions have a fundamental focus, that is, of providing a platform as a challenge whereby the motivation to do well at these competition inadvertently drives the participants to discover and use new knowledge. These formats, however, are constant instances of similar test arenas (i.e. the defined physical operational environment for the robot's operation) that is found in competition to competition. This raises a challenge to potential participants with various degrees of knowledge and ability. As these competitions are mainly patronised in academia, the participants are mostly undergraduates or postgraduates. These competition are used as a research and learning platform. However, the prerequisite for such participation is demanding as it requires that they have background knowledge in engineering to computer programming. That is, the entry point to become involved in this form of competition is too prohibitive to most. The current work presented here details a multiple format for competitive robots that provides continuity to allow participants from primary school ages to postgraduate researchers, in graduated phases. This allows participants to progress from one phase to the next as their technical knowledge and skill improves. 


\section{Background}

Competitive robots can be divided into three categories to include (1) intelligent autonomous robots, (2) remote controlled robots, and (3) passive or sympathetic (peripheral) robots. Each category provides different level of challenges to different age groups. These challenges provide specific problems for participants to solve or attempt to their best to overcome the challenges.

\subsection{Intelligent Autonomous Robots}

Intelligent autonomous robots are deployed in arenas to interact with objects or other robots to overcome specific problems. This includes soccer-playing robots and search and rescue (SAR) robots. Robots in this category employ exo-shells that resemble actual organic forms such as bipedal and quadpod creatures (e.g. Sony Aibo canine robots and Lynxmotion range of bipeal robots). Other forms are wheeled or track autonomous vehicles (e.g. Fischertechnik, Lego NXT and FIRA-based robots) [3, 4]. In order to address specific challenges such as scoring a goal in the opponent's goal, the autonomous robots must demonstrate a very high-level of intelligence. To accomplish this, computational intelligence methods need to be programmed into embedded microprocessor controllers, which further controls motors, sensors, servos and other type of actuators. Hence, a very high level of knowledge is required to build, program and operate robots in this category.

\subsection{Remote Controlled Robots}

Remote controlled robots within this category may be physically similar to intelligent autonomous robots. They may employ the same type of exo-shell and mechanics to resemble forms that would best help to overcome challenges within the competition. However, they do not demonstrate or have very little in-built intelligence. They are mostly tele-operated using wireless or tethered remote controllers. The primary purpose of this type of competition is to test and challenge the physical, mechanics and electronic aspects of the robots. Remote controlled robots are used in full-scale SAR urban test arenas and popular in competitive categories such as sumo-bot and battle-bots [5].

\subsection{Passive or Peripheral Robots}

Passive or peripheral robots neither demonstrate intelligence or are remote controlled. Their function is primarily to play the sympathetic role of ornamentation. With the exception of pre-programmed mobility and movement, it has very limited 'awareness'. Even though this may seem superfluous, their very appearance in the arena is inherent in a competition involving robots. Peripheral robots are employed in the dancing robots category in competition such as RoboCup Jr [2]. Categories such as this provide very young children with limited technical knowledge the opportunity to participate in robot competitions. 


\section{Multi Format Robot Search and Rescue (SAR) Arena}

The development of the multi format robot competition adopts SAR arena as it provides the best platform and potential for extension [10]. The purpose of a multi format SAR arena is to provide accessibility to all participants ranging from young age children to postgraduate researchers. Each category provides continuity from one phase to the next, in turn providing increasingly more challenging problems for the participants to overcome. In this way, participants are not required to have comprehensive prerequisites to participate in a robot competition. For the purpose of the multi format robot SAR arena competition, only intelligent autonomous robots are considered. The format begins as a 2-dimensional flat arena, subsequently upgrading to a 3dimensional arena and then finally to a problem-based arena.

\section{2-Dimensional Arena}

The 2-dimensional arena is based on the RoboCup Jr rescue robot format. Participants are to design, build and program an intelligent autonomous robot that could navigate across an arena following a pre-defined marked path to arrive at a pre-destined 'disaster' area to 'rescue' a victim. The arena is composed of $50 \times 50 \mathrm{~cm}$ flat surface platform tiles arranged edge-to-edge in a continuous path. The complexity depends on the age group of the participants. Fig. 1 shows an actual competition in progress, the 2-dimensional arena arranged in the desired path.
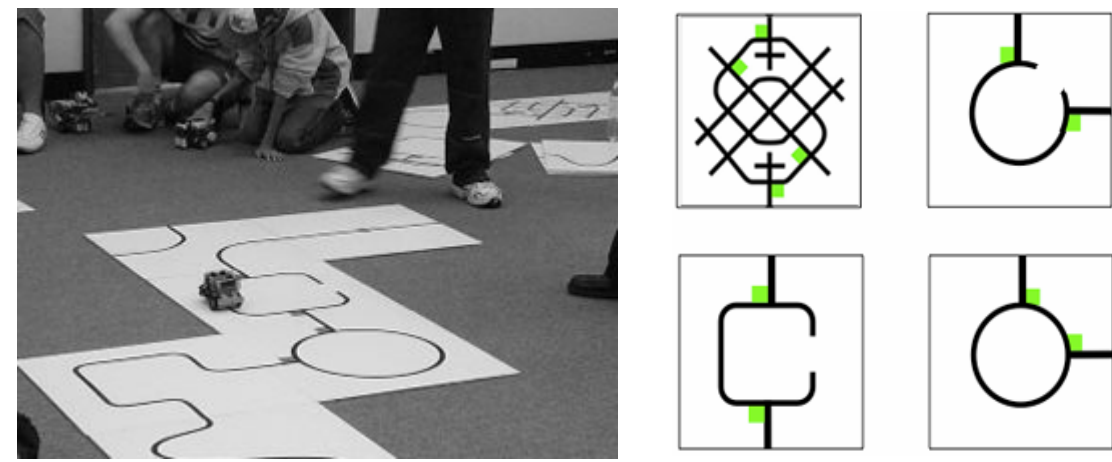

Fig. 1. 2-dimensional search and rescue robot arena based on RoboCup Jr (http://www. robocup.org/). Platform tiles are arranged edge-to-edge to provide a continuous path. Four samples from a possible pool of approximately $15-18$ different tiles are shown above. Tiles are designed with straight paths to increasingly complex paths.

In this category, the intention of the competition is to provide challenges to participants to demonstrate their competence in construction and programming. In most cases, the use of construction kits such as Lego NXT and Fischertechnik are commonplace. For more advanced participants, pre-fabricated embedded systems are used such as Arduino and Parallax Propeller microprocessors. In the advanced version of 
the 2-dimensional arena, participants are not only required to follow a pre-destined path to arrive at a destination, the robots must be sufficiently intelligent to avoid simple obstacles and slightly raised surfaces - a prelude to what is expected in the challenges expected in the follow up found in subsequent 3-dimensional arenas.

\section{3-Dimensional Arena}

\subsection{Entry Level Competition Arena}

The 3-dimensional arena is based on the 2-dimension version with added complexity. Obstacles in a variety of material is added to provide scaled-down version of possible real-life scenarios. Understandably, the 3-dimensional arena is a huge leap from the 2-dimensional version. Hence, in order to provide continuity in terms of gradual difficulties of the challenge, two version of the arena is employed. The first has a marked path (Fig. 2). The autonomous robot is only required to overcome the physical obstacles minimising the need to intelligently traverse in the arena. Autonomous navigation is a complex study in itself, therefore by reducing this requirement, the 3-dimensioanl arena is now made more accessible to participants with moderate background knowledge and skills.

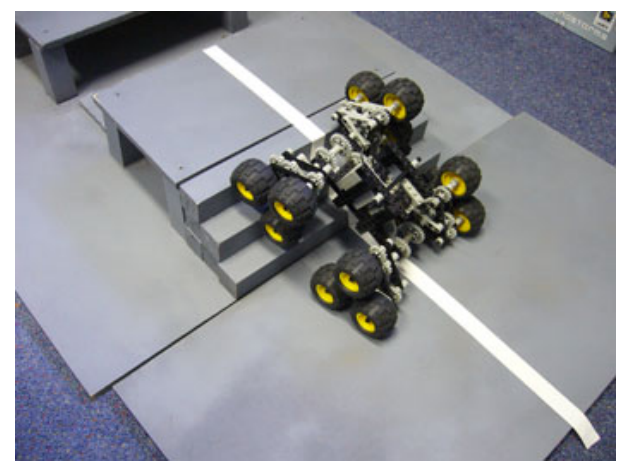

Fig. 2. 3-dimensional search and rescue robot arena. The entry-level category has a marked path to simplify the necessity to program complex navigational functionality into the scaled down SAR robots.

\subsection{Advanced Level Competition Arena}

The advance version of the 3-dimensional arena is similar to the entry-level arena without the marked path. Also, based on the possible geomorphic arrangement, the test arenas can be arranged in any configuration depending on the requirements of the competition.

The purpose of this category (entry and advanced level) is to provide challenges that allow participants to demonstrate their ability in not only constructing and developing advanced robots that are capable of overcoming physical obstacles, participants should also be able to demonstrate their programming skills. It is expected that 


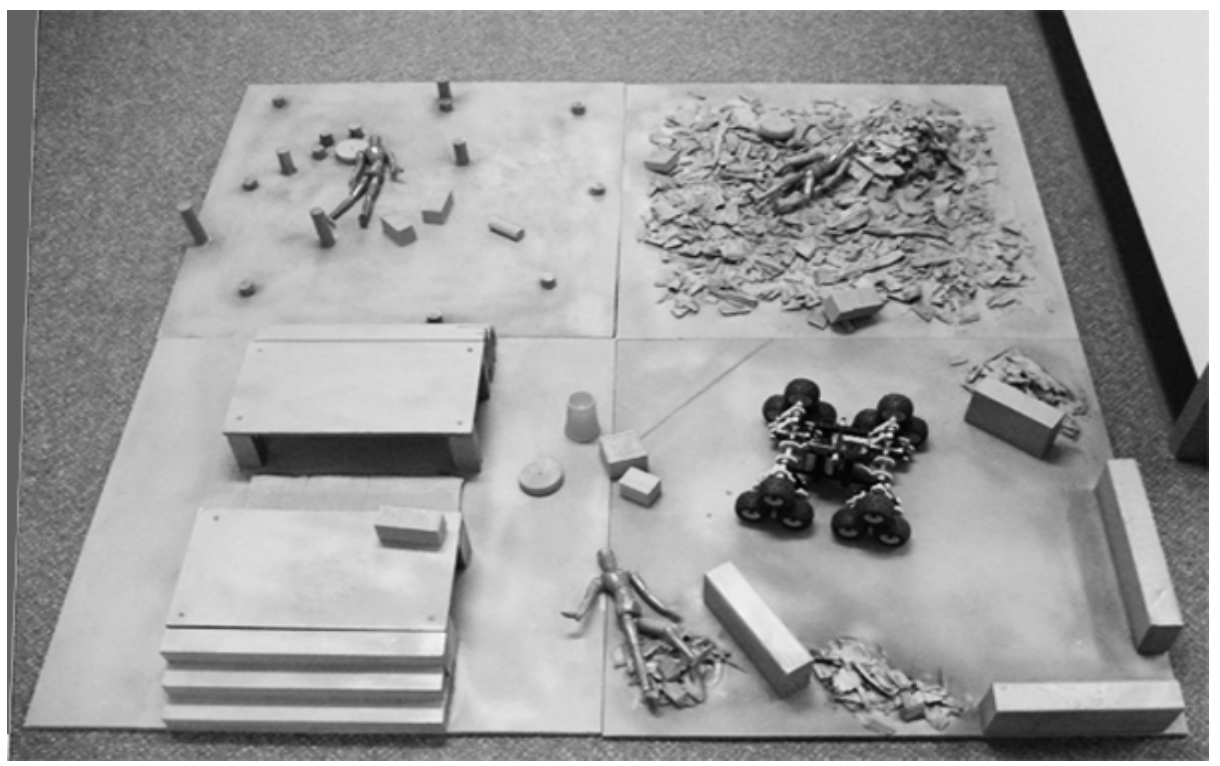

Fig. 3. 3-dimensional search and rescue robot arena. Four platform tiles arranged in a geomorphic configuration. The arena is populated with scaled-down effigies of victims, debris and moveable objects.

programming skills would require a high level of proficiency in intelligent computational methods [6].

The arena will be populated with effigies depicting victims in real life scenarios [7]. The goal of each SAR robots in the test arena is to locate, and if possible, retrieve the victims. Secondary goals for the robots are to map the area and provide environmental data to the human operators. Overall, the competition is to address each of the following requirements:

- The ability to assess a structure and assist in shoring it for increased stability.

- The ability to enter and travel around small confined spaces that cannot be accessed by humans or rescue dogs (to scale).

- The ability to carry supplies, such as extra oxygen tanks (to scale) and tools for rescuers to use.

- The ability to monitor the critical signs of a victim and provide first aid or food and water or both, until the victim can be removed from the area.

- The ability to process images quickly and extensively, often coupled with infrared sensing capabilities, which allow them to do a more complete survey of their surroundings.

- The ability to check and monitor different environmental factors, such as temperature and atmospheric gases continuously. This would allow them to provide early warning of problems that might otherwise go unseen by rescue workers until too late.

- The ability to detect harmful materials. These robots can be programmed to discover the hazardous material and inform rescuers or take recourse to neutralise it. 


\section{Problem Based Arena}

The problem based arena in robot competition is the outcome resulting from cumulative concepts inherited from the 2- and 3-dimensional arenas. The goal of the problem based arena is to progress into real life case studies modeled on its predecessors. Even though this may move away from the search and rescue principle inherent in such competitive robots, the technology and skills required remains unchanged. The purpose of this arena is to allow the participants to apply their expertise in a known problem area [9]. By deploying this as a competition, it inevitably support a solution based approach expeditiously. In the following experimental arena, an example is presented to show how the SAR multi format is applied to one of the many problem based arena representing real-life challenges.

\subsection{Problem Based Scenario}

Despite on-going attempts and current research to utilise intelligent systems technology to both provide advice on pest control strategies and to monitor mobile pests, the actual execution of the control and management task is ultimately manual driven and labour intensive [8]. In the attempt to substitute human labour with automated mechanised devices, autonomous mobile robots have been one of the most popular choices (Fig. 4). Ideally, robots are the mechanical-electronic embodiment of human functions. Even though this functionality has only been partially realised in specific challenges, the robots ability to perform task repetitively far outweighs many of its disadvantages. However, the utmost benefit of using autonomous robots over human labour is the minimising of risk and injury commonly encountered by its human counterpart.

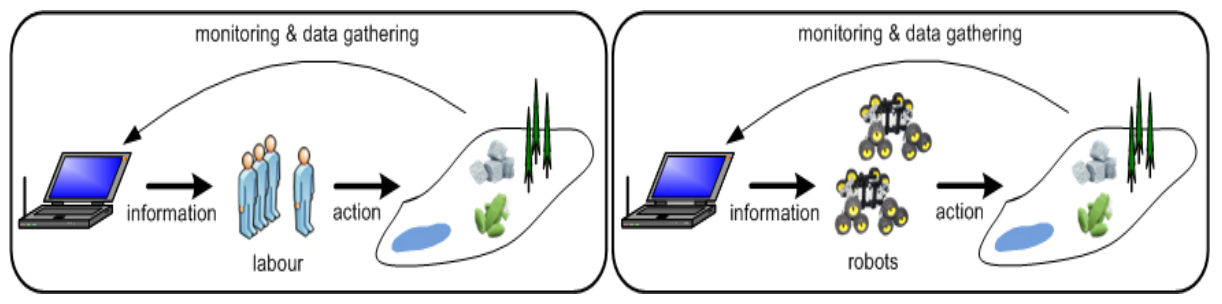

Fig. 4. Labour vs. Autonomous Robotics. Despite utilisation of computerised intelligent and monitoring systems, subsequent follow up action to control invasive pest is ultimately labour intensive. The capability to deploy autonomous robots to substitute human labour should be further investigated.

\subsection{The Challenge}

The SAR robot must be developed in a suitable operational environment. This environment is critical in facilitating the investigation, development and testing of functional components that comprises the hunter (i.e. SAR) robots. Fig. 5 illustrates the operational environment consisting of four entities and one staging area:

1. Hunter robots. This is the core component of the problem based arena.

2. Data collection station (for hunter robots and prey robots). 
3. Working models to mimic stationary threats, i.e. obstacles, water, debris as Prey Type T-1).

4. Working models to mimic mobile threats, i.e. cane toad (designated as Prey Type T-2). This can be quickly built using construction kits.

5. The staging area is a collection of test arenas that itself comprise of different obstacles to simulate natural habitat of T-1 and T-2. The test arenas are isometric and geomorphic, that is, it allows the arenas to be setup in different configurations to allow almost any type of habitat to be replicated economically. The segmented test arenas can also be conveniently stored away or transported.

Overall, the competition (i.e. investigation) is to address each of the following requirements:

1. Pioneering the concept of using a singular hunter robot type to seek and control multiple types of threats and prey.

2. The use of electro-mechanical devices to mimic pests in order to test the performance of hunter robots is in itself significant innovation. The ability to model known pests accurately contributes directly to the ability in identifying the actual pests.

3. The utilisation of test arenas to artificially simulate a known habitat is significant. The ability to simulate a habitat accurately contributes directly to the ability to extract features from background noise. That is, differentiating specific specie from its surroundings.

4. Improving and refining the hardware devices used in robots. This may include the development of better tactile sensors, actuators, power supply and efficient locomotion.

5. A robot is only as good as the software that controls it. A functioning robot that successfully addresses the challenges proposed in this project should inevitably lead to better software techniques such as artificial intelligence methods, image processing algorithm, smart software and software architecture.

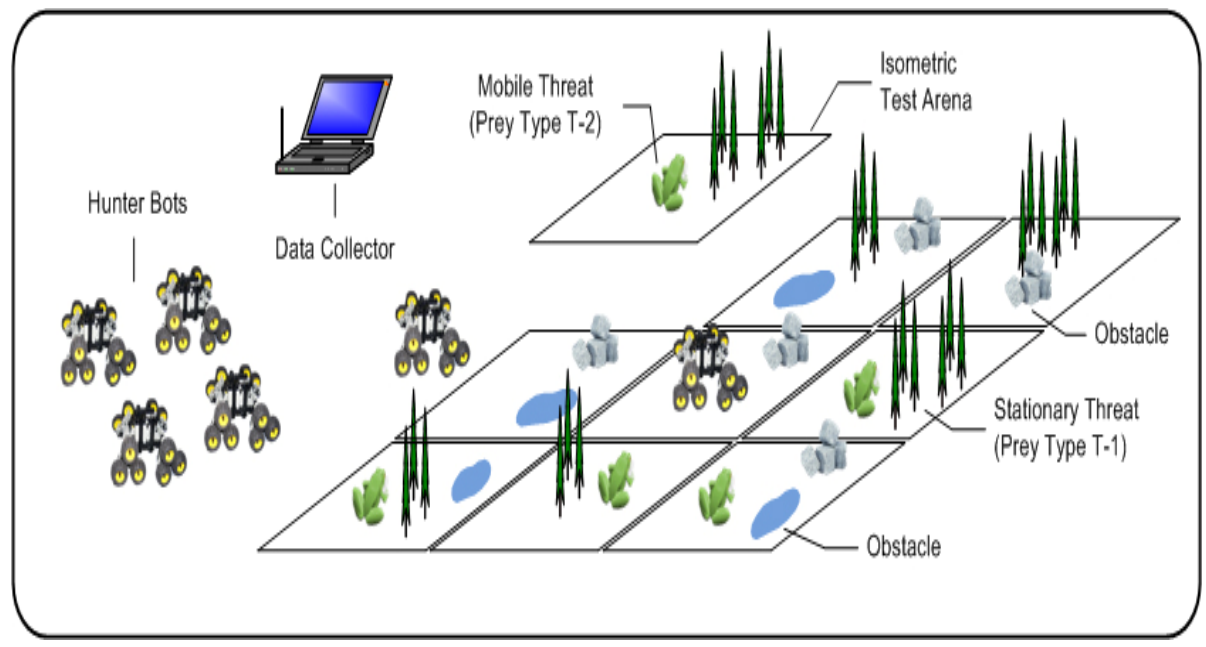

Fig. 5. Operational environment to facilitate the research, development and testing of hunter robots 


\section{Summary}

Current robot competition provides both students and researchers a platform to experiment with related technology and skills to solve challenging problems. However, the entry point for participation requires very demanding prerequisites of the participants. Even though robot competition is gaining popularity as a form of digital entertainment, it is however a very specialised domain catering to academia. This has presented on-going work in specifying, designing and developing a multiple format search and rescue robot to provide continuity for primary age participants to postgraduate researchers.

\section{References}

1. http: //www. fira.net/ (accessed on: May 10, 2010)

2. http: / /www. robocup.org/ (accessed on: May 10, 2010)

3. Tominaga, H., Onishi, Y., Hayashi, T., Yamasaki, T.: LEGO Robot Programming Exercise Support for Problem Solving Learning with Game Strategy Planning Tools. In: First IEEE International Workshop on Digital Game and Intelligent Toy Enhanced Learning (2007)

4. Asada, M., D’Andrea, R., Birk, A., Kitano, H., Veloso, M.M.: Robotics in Edutainment. In: IEEE International Conference on Robotics and Automation 2000, ICRA 2000 (2000)

5. http://www.battlebots.com/ (accessed on: May 10, 2010)

6. Kitano, H., Tadokoro, S., Noda, I., Matsubara, H., Takahashi, T., Shinjou, A., Shimada, S.: Robocup Rescue: Search and Rescue in Large-Scale Disasters as a Domain for Autonomous Agents Research. In: Proc. IEEE Conf. on Systems, Man, and Cybernetics, vol. 6, pp. 739-743 (1999)

7. Jacoff, A., Messina, E., Evans, J.: A Standard Test Course for Urban Search and Rescue Robots. In: Proc. of Performance Metrics for Intelligent Systems Workshop (2000)

8. Chiou, A., Yu, X.: A large-scale Agro decision support system: framework for (physical) fusion of a multi-input and multi-output hybrid system. In: Proc. the Third International Conference on Intelligent Sensors Sensor Networks and Information Processing, Melbourne (2007)

9. Chiou, A., Wynn, C.: Urban Search and Rescue Robots in Test Arenas: Scaled Modeling of Disasters to Test Intelligent Robot Prototyping. In: The Sixth International Conference on Ubiquitous Intelligence and Computing, Brisbane (2009)

10. Jacoff, A., Messina, E., Weiss, B.A., Tadokoro, S., Nakagawa, Y.: Test arenas and performance metrics for urban search and rescue robots. In: IEEE/RSJ International Conference (2003) 